\title{
Adult Onset Still's Disease Presenting with Acute Respiratory Distress Syndrome: Case Report and Review of the Literature
}

\author{
Anisha B. Dua ${ }^{*}$, , Augustine M. Manadan² and John P. Case ${ }^{2}$ \\ ${ }^{1}$ University of Chicago Medical Center, ${ }^{2}$ Rush University Medical Center and John H. Stroger, Jr. Hospital of Cook \\ County, USA
}

\begin{abstract}
Introduction: Adult-onset Still's disease (AOSD) is a systemic inflammatory disorder characterized by rash, leukocytosis, fevers, and arthralgias. Pulmonary involvement has been reported rarely in AOSD, but acute respiratory distress syndrome (ARDS) is extremely rare and potentially fatal and must be recognized as potential manifestation of underlying AOSD.
\end{abstract}

Methods: We present a case of AOSD manifested by ARDS and review the previously reported cases in Medline/Pub med.

Results: Including this case, 19 cases of AOSD complicated with ARDS have been reported in the literature.

Conclusions: It is important to recognize ARDS as a manifestation of AOSD so that proper diagnostic and therapeutic management can be initiated promptly.

Keywords: Still's Disease, ARDS.

\section{INTRODUCTION}

Adult onset Still's disease (AOSD) is a systemic inflammatory disorder that is related to systemic-onset juvenile idiopathic arthritis. Like the pediatric disease, it is characterized by spiking fevers, arthralgias, salmon colored rash, leukocytosis, sore throat, cytopenias and hyperferritinemia. Severe systemic manifestations associated with AOSD include disseminated intravascular coagulation (DIC), macrophage activation syndrome (MAS), and hepatic failure. Pulmonary involvement, usually pleuritis, is a recognized manifestation of AOSD. Parenchymal lung disease and acute respiratory distress syndrome (ARDS) are rarer pulmonary manifestations of AOSD. We report a case of a 26 year old female with AOSD presenting with ARDS complicated by MAS.

\section{METHODS}

First, a patient with AOSD and ARDS is described and the clinical, laboratory, and radiologic findings are reported. Second, Medline/Pub med was also searched for associations between ARDS, SIRS, and AOSD using combinations of the keywords "acute respiratory distress syndrome", "ARDS", "systemic inflammatory response syndrome", "SIRS", "adult onset Stills disease", and "Stills disease". A search was also done using OVID SP with these same keywords, and relevant citations from reported cases were reviewed.

*Address correspondence to this author at the University of Chicago Medical Center, Department of Rheumatology, 5800 S. Maryland Ave, MC0930, Room \#N005, Chicago, IL 60637, USA; Tel: 773-702-8702;

E-mail: adua@medicine.bsd.uchicago.edu

\section{RESULTS}

\section{Case Report}

A 26-year-old African-American female with a history of type 2 diabetes mellitus presented to her primary care physician with sore throat and cough. She was noted to have a "viral exanthem", and was started on amoxicillinclavulanate. Her symptoms did not improve and she went to the emergency department (ED) for shortness of breath, cough and myalgias. Her vital signs were as follows: temperature $36.7 \mathrm{C}$, pulse rate 112 , respiratory rate 18 , and blood pressure 100/56 mm Hg. Physical examination was notable for coarse breath sounds bilaterally over lung fields with scattered crackles at the bases and tachycardia. Radiograph of the chest showed bilateral interstitial infiltrates suggestive of ARDS. Arterial blood gas (ABG) showed pH 7.28, pCO2 $38 \mathrm{mmHg}$ and pO2 $58 \mathrm{mmHg}$. Her respiratory status worsened and she underwent endotracheal intubation requiring ventilator support, and was started on broad-spectrum antibiotics for presumed pneumonia.

Two days after admission to the intensive care unit (ICU), the patient developed daily spiking fevers up to 40.0 C. She became more difficult to oxygenate with increasing ventilator requirements. She became hypotensive to 70/49 $\mathrm{mmHg}$ and required vasopressors. Antibiotic therapy was broadened and changed multiple times, including treatment with vancomycin, ceftriaxone, azithromycin, piperacillin/ tazobactam, imipenem, metronidazole and fluconazole, without any clinical improvement.

Laboratory analysis included an initial white blood cell (WBC) count of $20 \times 10^{3} / \mathrm{mm}^{3}$ (which increased to $49 \mathrm{x}$ $10^{3} / \mathrm{mm}^{3}$ within one week) with $91.8 \%$ neutrophils. 
Hemoglobin $(\mathrm{Hb})$ was initially $10 \mathrm{~g} / \mathrm{dL}$ (and dropped to 8.3 $\mathrm{g} / \mathrm{dL}$ within one week), and platelet count 407,000/mL. Aspartate aminotransferase (AST) was $88 \mathrm{IU} / \mathrm{L}$, alanine aminotransferase (ALT) $44 \mathrm{IU} / \mathrm{L}$, and lactate dehydrogenase (LDH) 530 units/L. Autoimmune panel was negative for anti-nuclear antibodies (ANA) and rheumatoid factor (RF). C-reactive protein (CRP) was elevated at $7.19 \mathrm{nmol} / \mathrm{L}$ and erythrocyte sedimentation rate (ESR) was $39 \mathrm{~mm} / \mathrm{h}$. The ferritin level was $4,022 \mathrm{ng} / \mathrm{mL}$.

The patient underwent further extensive evaluation during the first ten days of her admission. All cultures during fever spikes were negative, including seven sets of blood cultures. Computerized axial tomography (CT) scans of her neck and sinus were unremarkable. CT of the chest revealed bilateral pleural effusions and diffuse, patchy airspace opacities and interstitial infiltrates. She underwent bronchoscopy which was negative for bacterial, viral (including cytomegalovirus) or fungal infection. Hepatitis panel, urine legionella, serum histoplasma antigen, cryptococcal antigen, Clostridium dificile toxin, and acid fast bacilli were all negative. Transthoracic echocardiogram was normal with an ejection fraction of 55-65\%. Bone marrow biopsy and flow cytometry were normal.

She was diagnosed with AOSD based on fulfillment of the Yamaguchi criteria with major criteria of fevers lasting greater than one week, leukocytosis with neutrophilia, and minor criteria of abnormal liver enzymes, sore throat, and negative RF and ANA [1]. In addition, she had hyperferritinemia and no evidence of infection or malignancy after extensive investigation. She was started on methylprednisolone $60 \mathrm{mg}$ intravenously (IV) every 6 hours on day 13 of her hospital admission, and the next day, her maximum temperature improved to $38.2 \mathrm{C}$, down from 39.4 C. On day 16 she was given pulse methylprednisolone $1000 \mathrm{mg}$ daily for 3 days, followed by prednisone $60 \mathrm{mg}$ daily. She remained afebrile until her discharge on hospital day 19. She was weaned off vasopressors, antibiotics were discontinued, mental status was at baseline, and WBC count improved prior to discharge. She had developed an ICU polyneuropathy during her hospital course and required tracheostomy and transfer to a ventilator facility for weaning from ventilator support. At the ventilator facility she was continued on prednisone $60 \mathrm{mg}$ daily except for one missed dose of prednisone the week prior to re-admission.

Two weeks after her discharge, she was re-admitted to the ICU with spiking fevers to $39.4 \mathrm{C}$, and again, broadspectrum antibiotic therapy with vancomycin, tobramycin, and metronidazole was initiated. Two days after admission, she had a seizure followed by hypotension to $87 / 43 \mathrm{mmHg}$ and required vasopressor support. Seizures were treated with lorazepam and phenytoin. Imipenem, metoclopramide, and metronizadole were discontinued, and meropenem and oral vancomycin were initiated. Laboratory testing revealed a WBC count of $15.1 \times 10^{3} / \mathrm{mm}^{3}, \mathrm{Hb} 6.9 \mathrm{~g} / \mathrm{dL}$, platelets 61,000/mL, AST $627 \mathrm{IU} / \mathrm{L}$, ALT 507 IU/L, LDH 2,080 units/L, ferritin 110,065 ng/mL, fibrinogen $170 \mathrm{~g} / \mathrm{L}$, normal coagulation studies and ESR $16 \mathrm{~mm} / \mathrm{h}$.

She continued to have seizure activity, electromyogram showed moderate to severe axonal sensorimotor polyneuropathy, and electroencephalogram revealed epileptiform activity over the right parietal region and prominent sharp waves over bilateral temporal regions. Magnetic resonance imaging of the brain revealed multifocal stenosis of bilateral posterior cerebral arteries and a segment of the left anterior cerebral arteries. CT chest showed bilateral large pleural effusions with consolidation and airspace opacities consistent with ARDS. The patient was started on methylprednisolone $1000 \mathrm{mg}$ IV daily for three days followed by prednisone $60 \mathrm{mg}$ daily and cyclosporine $3 \mathrm{mg} / \mathrm{kg} /$ day for presumed macrophage activation syndrome (MAS). Because of her continued deteriorating course, a repeat bone marrow biopsy was done over two weeks after cyclosporine therapy was begun and showed positive staining for cytomegalovirius (CMV) without evidence for hemophagocytosis. CD 163 staining to look for activated histiocytes was not performed. In addition, the cerebrospinal fluid (CSF) was positive for CMV by PCR and the patient was started on gancyclovir. The patient's presentation was felt to meet criteria for CMV infection and secondary hemophagocytic syndrome despite bone marrow absence of true hemophagocytosis. The patient died despite treatment and supportive care measures six weeks after her initial presentation with ARDS and AOSD.

\section{LITERATURE REVIEW}

A Medline search yielded a total of 12 previously reported cases of ARDS associated with AOSD. Two of these case reports were in Japanese and French, and abstracts were unavailable. Of the other 10 , references led to three more articles, one with three reported cases of AOSD with ARDS, one with two reported cases, and the other, a single case report, for a total of 18 reported cases. By description, all 16 accessible cases reviewed met the Yamaguchi criteria for AOSD. The patients ranged from 17-71 years of age. Fever and leukocytosis was present in all cases. Reported ferritin values ranged from 103-42,600 ng/mL. All patients were treated with corticosteroids and a few had additional treatments with methotrexate, azathioprine, cyclosporine, cyclophosphamide, and intravenous immunoglobulin (Table 1).

\section{DISCUSSION}

AOSD is characterized by high spiking fevers, rash, sore throat, and hyperferritinemia. Multiple sets of diagnostic criteria for AOSD have been proposed, but the Yamaguchi criteria have the highest sensitivity of $93.5 \%$ [1]. Our patient fulfilled the Yamaguchi criteria with spiking fevers, leukocytosis with neutrophila, sore throat, lymphadenopathy, abnormal liver enzymes, and negative infectious, rheumatic, and malignancy evaluations.

After her admission, she rapidly developed ARDS. Viral cultures of bronchoscopic alveolar lavage fluid, tested during her first admission were negative for CMV. This culture has a reported sensitivity of $100 \%$ and specificity of $70 \%$ [2]. Her initial presentation with ARDS is thus unlikely to be attributable to CMV pneumonia, and all other infectious workup was negative. However, on her second admission, after treatment with high dose corticosteroids for 3 weeks, she was found to have positive $\mathrm{CMV}$ staining on bone marrow biopsy and in the CSF by PCR. We concluded that 
Table 1. Case Reports of Acute Respiratory Distress Syndrome associated with Adult-Onset Still's Disease

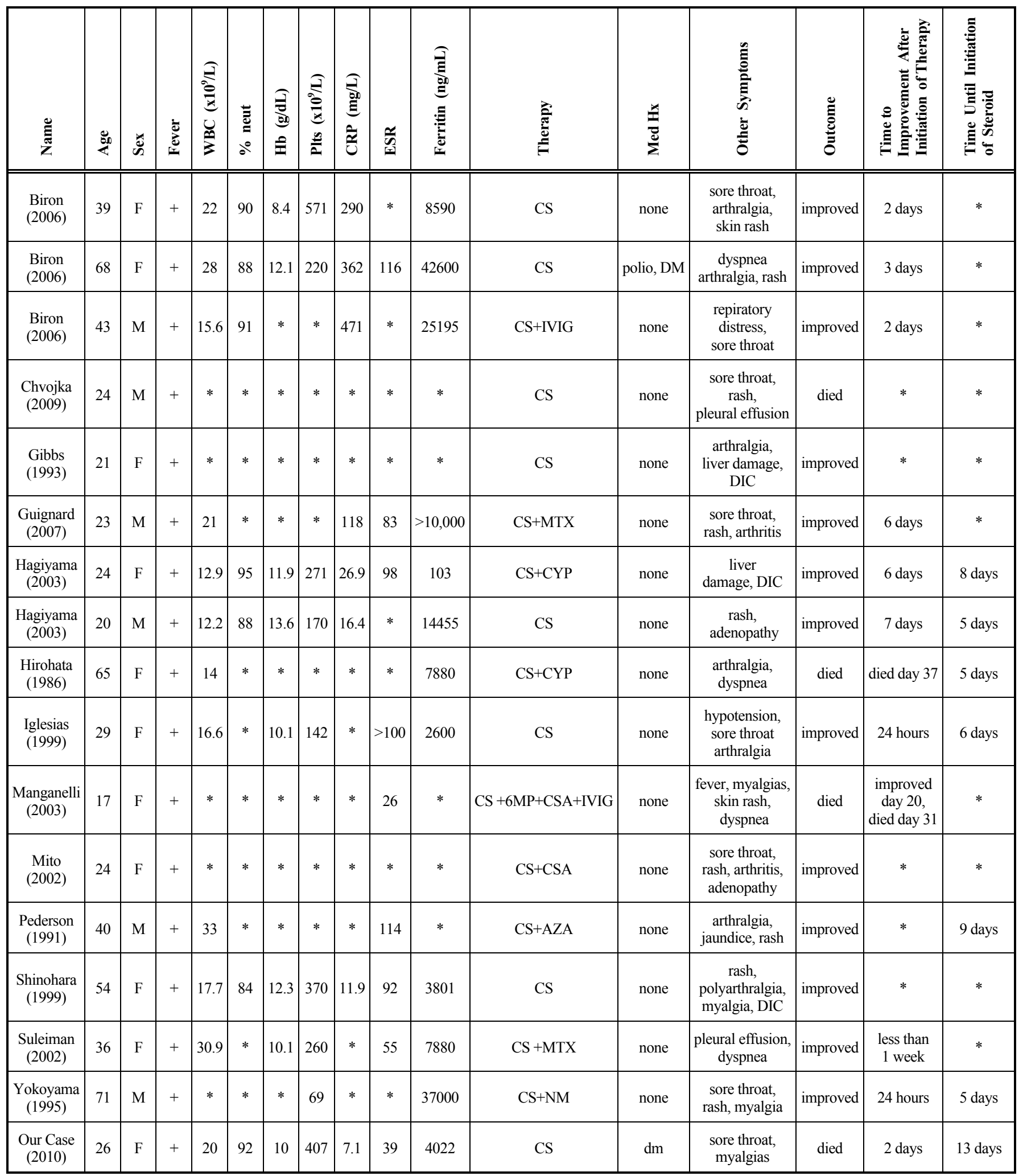

*-Data/values not reported or unavailable.

Abbreviations: $\mathrm{F}=$ female, $\mathrm{M}=$ male, $\mathrm{WBC}=$ white blood cell count, neut= neutrophil, $\mathrm{Plts}=$ Platelets, $\mathrm{CRP}=\mathrm{C}$ reactive protein, $\mathrm{gly}=\mathrm{glycosolated}, \mathrm{Med}=\mathrm{medical}, \mathrm{Hx}=\mathrm{history}, \mathrm{CP}=$ Chest pain, $\mathrm{SOB}=$ shortness of breath.

DIC $=$ disseminated intravascular coagulation, $\mathrm{DM}=$ diabetes mellitus, $\mathrm{CS}=$ corticosteroids, $\mathrm{CSA}=$ cyclosporine, $\mathrm{CYP}=$ cyclophosphamide, $\mathrm{IVIG}=$ intravenous immunoglobulin, MTX $=$ methotrexate, $\mathrm{AZA}=$ azathioprine

6-MP $=6$ mercaptopurine, $\mathrm{NM}=$ nafamostat mesilate.

Key for Table 1: Abbreviations: $\mathrm{F}=$ female, $\mathrm{M}=$ male, $\mathrm{WBC}=$ white blood cell count, neut $=$ neutrophil, $\mathrm{Plts}=$ Platelets, $\mathrm{CRP}=\mathrm{C}$ reactive protein, gly $=$ glycosolated, $\mathrm{Med}=$ medical, $\mathrm{Hx}=$ history, $\mathrm{CP}=$ Chest pain, $\mathrm{SOB}=$ shortness of breath, $\mathrm{DIC}=$ disseminated intravascular coagulation, $\mathrm{DM}=$ diabetes mellitus, $\mathrm{CS}=$ corticosteroids, $\mathrm{CSA}=$ cyclosporine, $\mathrm{CYP}=$ cyclophosphamide, IVIG = intravenous immunoglobulin, $\mathrm{MTX}=$ methotrexate, $\mathrm{AZA}=$ azathioprine, 6 -MP $=6$ mercaptopurine, $\mathrm{NM}=$ nafamostat mesilate. 
disseminated CMV infection complicated the immunosuppressive therapy and resulted in death 6 weeks after her initial presentation. The poor outcome in our patient and many others in the literature can hopefully be improved by early recognition and diagnosis of AOSD in those ARDS patients not responding to antibiotic therapy. It is also critical to re-evaluate for infectious causes if the clinical course declines after initiation of immunosuppressive therapy.

Pulmonary involvement in AOSD has been reported in multiple series, but ARDS has only been described in case reports [3-11]. Pleuritis is the most common pulmonary manifestation of AOSD, ranging from $12 \%-53 \%$ in various studies [12]. A review of pulmonary involvement in AOSD found that lung involvement was present ranging from $6 \%-53 \%$ of patients, most commonly pleuritis, followed by pleural effusions [13].

Severe pulmonary involvement, including pleuritis and interstitial pneumonia in AOSD, is associated with a poor prognosis. A retrospective analysis of 61 patients by Zeng et al. [12] found that $11.5 \%$ had interstitial pneumonia on CT. Six of the 61 patients in this series died, and 4 died from pneumonia/respiratory failure. Pulmonary involvement was found to be a poor prognostic sign in this study.

There are 18 case reports in the literature of AOSD manifesting with ARDS, and the 16 reports obtained have similar clinical and serologic findings to our patient [3-11] (Table 1). These cases were initially treated as infectious pneumonia with broad spectrum antibiotics, with subsequent respiratory decompensation and intubation. Antibiotics were discontinued in all cases, and followed by initiation of high dose methylprednisolone therapy. In most case reports, patients responded well clinically and serologically to high dose corticosteroid therapy within hours to days. In the reports by Hirohata [3], Manganelli [10], and Chvojka [11], the patients had AOSD complicated by ARDS, failed corticosteroid therapy and were subsequently treated with other immunosuppressive agents, but eventually died. Imaging findings of ARDS in these case reports were described as dense, diffuse, bilateral infiltrates, without any clear differentiating features between ARDS associated with infectious causes or other autoimmune diseases.

Early recognition of ARDS in patients with AOSD is clinically difficult, but important because it can lead clinicians to initiate therapy with corticosteroids. After treatment with methylprednisolone, patients had decrease in ferritin levels as well as improvement in respiratory and clinical status. In all the reviewed literature, as well as in our case, patients were initially managed with antibiotic therapy for ARDS. The subsequent diagnosis of AOSD complicated by ARDS led to a change in management.

Although our patient's bone marrow biopsy was negative for hemophagocytosis, her clinical features were consistent with MAS including fevers, thrombocytopenia, hyperferritinemia, transaminitis, anemia, and CNS manifestations. Prevalence of MAS in AOSD ranges from 11-15\%, and complications of pleuritis and ARDS were present in more than one third of patients who had MAS complicating AOSD [14].

The case presented here illustrates the severe presentation of AOSD with ARDS and its response to high dose corticosteroid therapy. Our patient presented with symptoms consistent with AOSD, but early classification and diagnosis was critical to guiding therapy. The diagnosis of AOSD should be considered in the face of ARDS when a patient is not responding to infectious therapy and other clinically compatible manifestations of AOSD are present, including spiking fevers and high ferritin levels. Early recognition of AOSD can lead to prompt initiation of appropriate therapy.

\section{CONFLICT OF INTEREST}

The authors declare that there are no conflicts of interest.

\section{ACKNOWLEDGEMENTS}

Declared none.

\section{REFERENCES}

[1] Masson C, Le Loet X, Liote F, et al. Comparative Study of 6 types of Criteria in Adult Still's Disease. J Rheumatol 1996; 23:495-7.

[2] Paradis IL, Grgurich WF, Dummer JS, et al. Rapid detection of cytomegalovirus pneumonia from lung lavage cells. Am Rev Respir Dis 1988; 138 (3): 697-702.

[3] Hirohata, S. Adult stills disease complicated with adult respiratory distress. Arch Int Med 1986; 146: 2409.

[4] Iglesias J, Sathiraju S, Marik PE. Severe systemic inflammatory response syndrome with shock and ARDS resulting from stills disease. Chest 1999; 115: $1738-40$.

[5] Suleiman M, Wolfovitz E, Boulman N, et al. Adult onset stills disease as a cause of ards and acute respiratory failure. Scand J Rheumatol 2002; 31 (3): 181-3.

[6] Biron Charlotte, Chambellan A, Agard C, et al. Acute Respiratory Failure Revealing Adult onset Stills disease. Clin Rheumatol 2006; 25: 766-68.

[7] Shinohara T, Hidaka T, Matsuki Y, et al. Calcinosis cutis and intestinal pseudo-obstruction in a patient with adult onset Still's disease associated with recurrent relapses of disordered coagulopathy. Internal Med 1999; 38 (6): 516-20.

[8] Hagiyama H, Koike R, Nagasaka K, et al. Two cases of acute respiratory distress syndrome resulting from adult-onset Still's disease. Med Rheumatol 2003; 13: 76-80.

[9] Guignard S, Dien G, Dougados M. Severe systemic inflammatory response syndrome in a patient with adult onset Still's disease treated with anti-IL1 drug anakinra: a case report. Clin Exp Rheumatology 2007; 25: 758-59.

[10] Manganelli P, Zuccoli P, Fietta P. Adult-onset Still's disease with respiratory distress syndrome, polyserositis and disseminated intravascular coagulation: a case with a fatal outcome. Clin Exp Rhematol 2013; 21: 139.

[11] Chvojka J, Krouzecky A, Radej J, et al. 24-year old male with fever, multi-organ dysfunction and fast progressing ARDS. Vnitr Lek 2009; 55 (10): 991-4.

[12] Zeng T, Zou Yu-Qiong, Wu Mei-Fang, et al. Clinical Features and Prognosis of Adult-Onset Still's Disease: 61 Cases from China. JCR 2009; 36: 1026-31.

[13] Cheema GS, Quismorio FP Jr. Pulmonary Involvement in Adult-Onset Still's Disease. Curr Opin Pulm Med 1999; 5: 305.

[14] Artlet JB, D Le Thi Huong, A Marinho, et al. Reactive Haemophagocytic Syndrome in Adult-onset Still's disease: a report of six patients and a review of the literature. Ann Rheum Dis 2006; 65: 1596-601. 